\title{
Advances in the Unified Theory of the Rotation of the Nonrigid Earth
}

\author{
Juan Getino \\ Grupo de Mecánica Celeste, Facultad de Ciencias, Valladolid, Spain
}

José M. Ferrándiz

Dept. Applied Mathematics, University of Alicante, Alicante, Spain

\section{Introduction}

Investigations of nonrigid-Earth rotation usually rely on the convolution of a transfer function with a rigid-Earth solution. A possible alternative is to develop a unified theory to encompass in only one body both rigid and nonrigid effects. It is represented in the Hamiltonian theory by Getino and Ferrándiz (GF). This theory combines the convenience of being a really analytical theory with good practical performance, since the residuals with respect to observations are also at the same \pm 1 mas level as the present IERS series.

We report on more recent results we obtained in the last year, not collected in the "Considerations" paper of the WGN (joint IAU/IUGG Working Group on Nutations).

\section{General features of GF theory}

The more relevant features of the GF theory are: 1) It is a unified and selfconsistent theory: rigid and nonrigid effects are derived from a unique Hamiltonian function. 2) It is enterely analytical: Lunar and planetary solutions being taken from analytical ephemerides (ELP2000, VSOP87). The solutions are obtained through a perturbation method (Hori's). 3) Parameters appearing in the analytical solution are global, and can be determined by numerical integration over the Earth's interior or by fitting the series to observations. 4) A general Hamiltonian formalism is used. We point out the computational advantage, especially to deal with second order (nonlinear) perturbations. 5) Precession and nutation can be treated jointly. Formulas for the precession of the nonrigid Earth can be derived from the secular part of the same Hamiltonian.

\section{Present stage of development}

The main part of the theory considers a three-layer Earth composed of mantle, Fluid Outer Core (FOC) and Solid Inner Core (SIC). Dissipation at the two boundaries is modelled similar to Sasao et al. (1980), taking into account both viscous and electromagnetic coupling. Elasticity is treated along the lines of Takeuchi, followed by Jeffreys and Vicente, and complemented with an elastic 


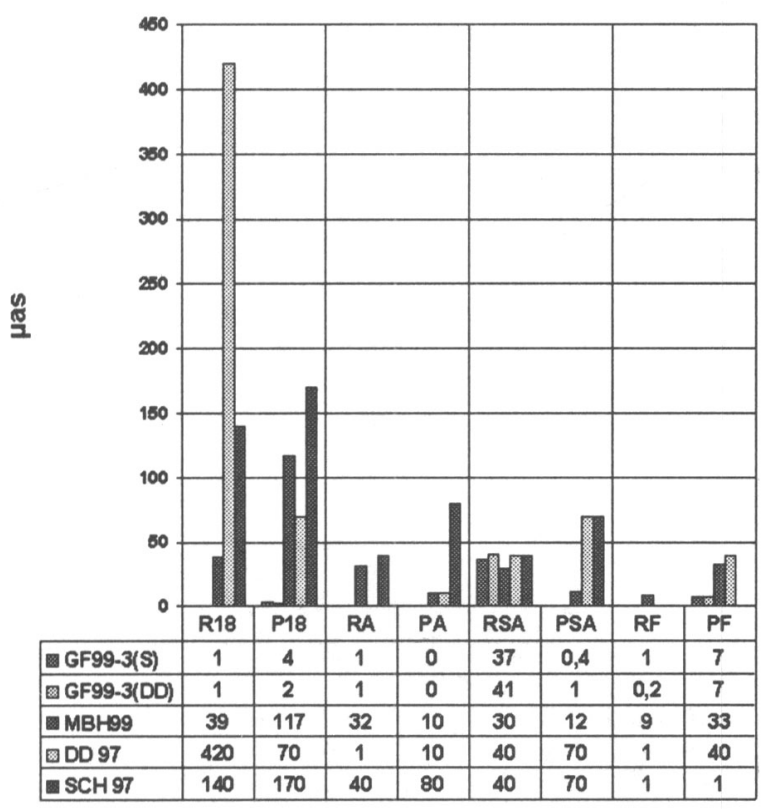

Figure 1. Absolute value of deviations with respect to IERS96. Inphase terms.

delay in the response. Nonrigid second order perturbations (in the sense of perturbation theory, that are quadratic in the dynamical ellipticity) have been partially derived. These terms cannot be found by the use of the transfer function approach that is essentially linear with respect to that perturbation parameter. High-frequency terms have been derived for the nonrigid model, corresponding to triaxiality (EGS99) and third and forth order harmonics. Analytical expressions for secular mixed terms are also already available (EGS2000). Nonrigid contributions from planetary origins have been computed in part, and are not negligible. The same is valid for the additional tidal potential. Analytical and numerical partial derivatives with respect to all the parameters are also available.

\section{Accuracy performance}

In Figures 1 and 2, prograde and retrograde amplitudes of the main terms provided by the series GF99-3 by Getino and Ferrándiz are compared with those of Mathews, Buffet and Herring 1999 (MBH99), Dehant and Defraigne 1997 (DD97) and Schastok 1997 (SCH97), showing the absolute value of respective deviations with respect to IERS96. Model GF99-3(S) includes the oceanic corrections given by Schastok (1997), while GF99-3(DD) uses the oceanic corrections provided by Dehant and Defraigne (1997).

Time domain deviations of GF99-3 series with respect to IERS96 are presented in Figure 3 for longitude $(\Delta \psi \cdot \sin \varepsilon)$ and Figure 4 for obliquity $(\Delta \varepsilon)$. Next we compare the time domain deviations of GF99-3 and IERS96 with respect to the observations USN09903. Deviations corresponding to our series GF99-3 are 


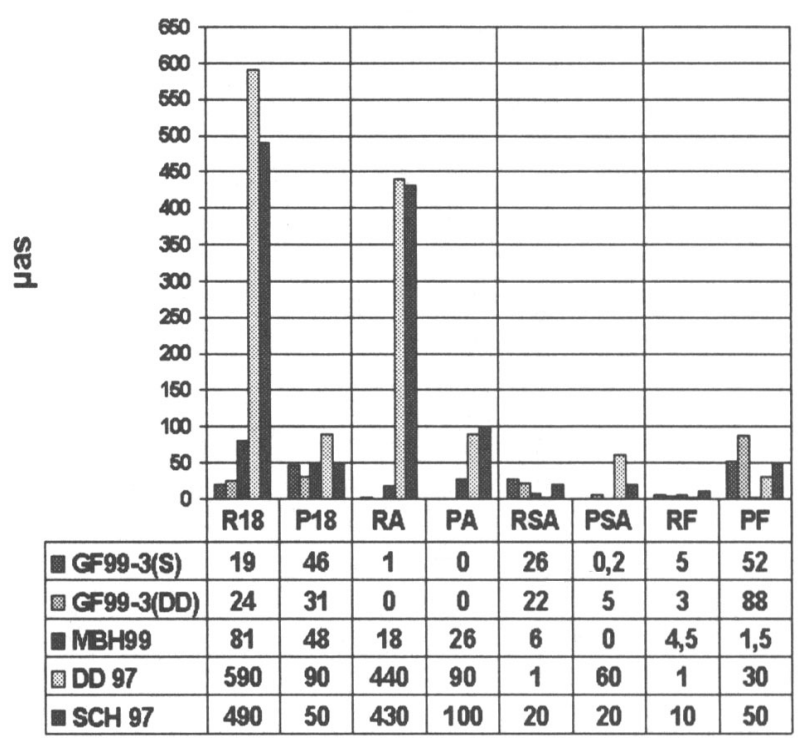

Figure 2. Absolute value of deviations with respect to IERS96. Outof-phase terms.

marked with a symbol "+", and those of the IERS are marked with an "o". Figure 5 shows longitude $(\Delta \psi)$ and Figure 6 obliquity $(\Delta \varepsilon)$.

\section{Examples of new nonrigid contributions}

For the sake of brevity we present only some few terms.

1.- FCN resonance effect on Venus's direct perturbation: Main terms in longitude $(\mu a s): \Delta \psi=-113 \cos \left(2 L_{V e}-4 L_{E}+2 h\right)-82 \sin \left(2 L_{V e}-4 L_{E}+2 h\right)+$ $75 \sin \left(L_{V e}-L_{E}+h\right)$.

2.- Second order perturbation: main effect of FCN resonance on Oppolzer terms $(\mu a s): \Delta \psi=23 \sin (\Omega)+27 \sin (2 \Omega)$.

3.- Tidal potential: main contributions ( $\mu a s) \Delta \varepsilon=163 \cos (\Omega)$.

4.- Semidiurnal nonrigid main contributions $(\mu a s) \Delta \psi=-3 \sin (2 \phi-2 F-$ $2 \Omega)+4 \sin (2 \phi)$.

\section{Conclusions and suggestions}

Nowadays we are not forced to decide on choosing just one rigid Earth theory and one transfer function, because there is an alternative option available, namely to choose the GF unified theory to encompass in only one body both rigid and nonrigid effects. In our opinion, future IAU Resolutions concerning nutations should be aware of the existence of the unified Hamiltonian theory of the nonrigid Earth, and not restrict themselves to the several rigid Earth theory + transfer 


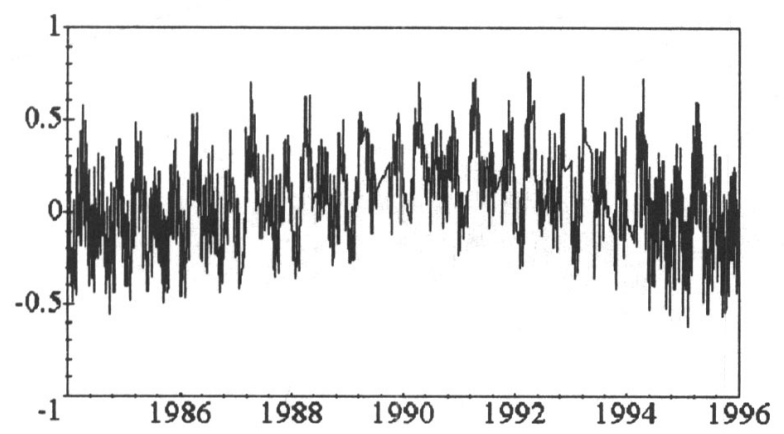

Figure 3. Time domain deviations of GF99-3: $\Delta \psi \cdot \sin \varepsilon$.

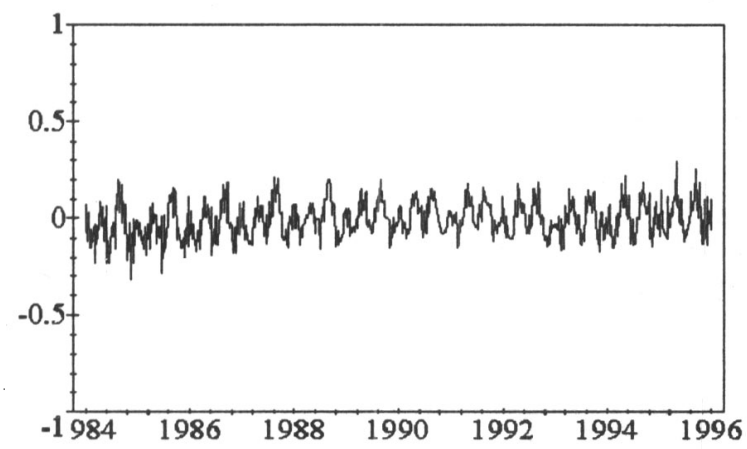

Figure 4. Time domain deviations of GF99-3: $\Delta \varepsilon$.

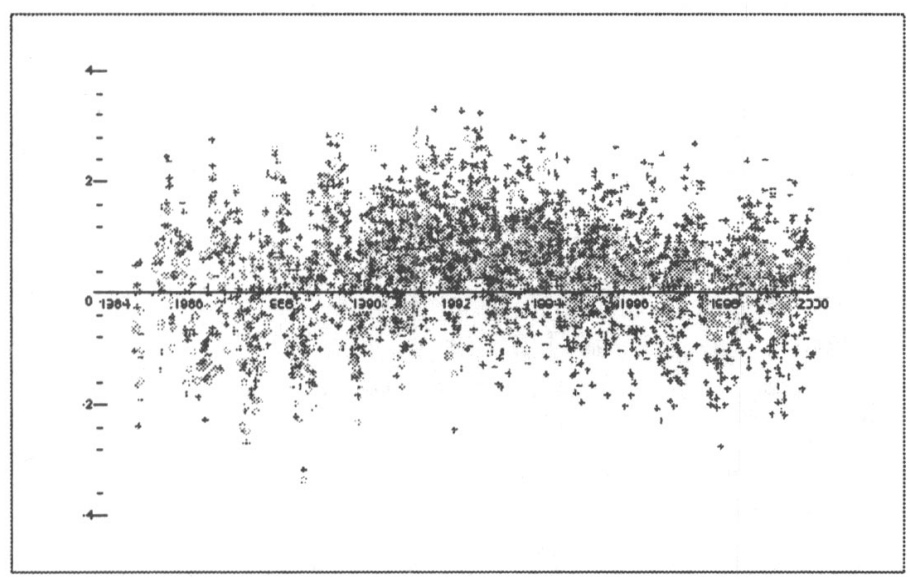

Figure 5. Time domain deviations with respect to observations: Longitude $(\Delta \psi)$. 


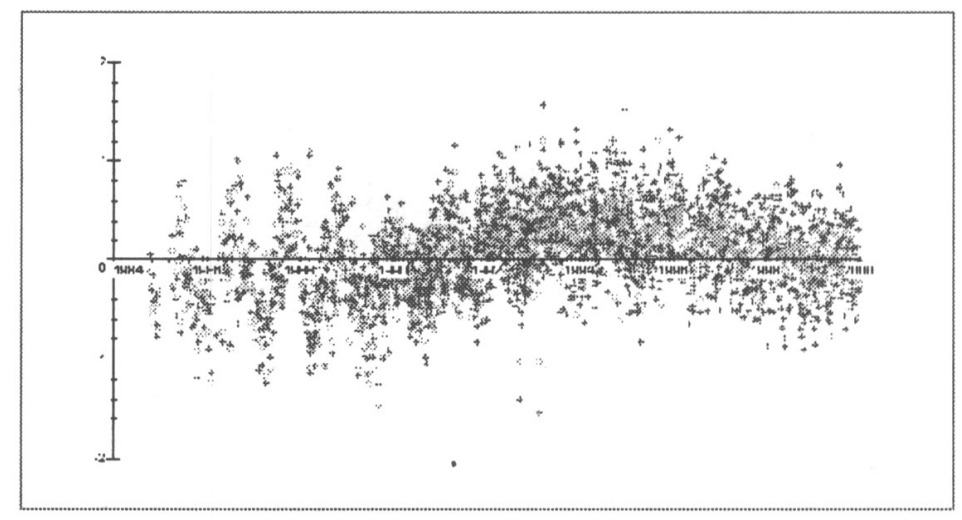

Figure 6. Time domain deviations with respect to observations: Obliquity $(\Delta \varepsilon)$.

function approaches. The reason is that the GF theory shows more capabilities than any other method, including state-of-the-art level of accuracy, complete consistency and ability to derive second order and other nonrigid corrections near or up to the microarcsecond level for each frequency. The latter is especially important for future improvements, since they are not likely to assume that the large discrepancy between all theories and observations (measured in terms of sigmas) is mainly due to chaotic dynamics and that we have reached the limits of predictability.

We have asked also the WGN to consider the possibility of our Unified Theory to play a role in the eventual adoption by the WGN of a new nonrigid Earth theory. The requirements of a new theory closer to observations than IAU 1980 may be covered by the combination of 1) a main analytical part, the Hamiltonian theory for the solid, nonrigid Earth described above, including partial derivatives and nonlinear high-order perturbations, 2) high-frequency and planetary terms, that should become more relevant in the future and should be derived from a nonrigid model at the 1-mas truncation level, and 3) some additional corrections corresponding, for instance, to the more well-known effect of outer geophysical fluids, at least the oceans.

Note this possibility is not in opposition to using successive IERS models to fit better the observations, like IERS' 96 or MHB2000. Coexistence of a theory and a computational model has been successful and stimulated advances for some years. In our opinion, a 'theory' should provide a solution as accurate and descriptive as possible to a physical model and as close as possible to the actual problem, whereas a 'computational model' should provide predictions as close as possible to observations even though the underlying physical situation is not completely understood (as happens in polar motion prediction by nonlinear dynamical techniques)

Acknowledgments. This work has been partially supported by Spanish Projects CICYT, ESP97-1816-C04-02, and JCYL, VA11/99. 


\section{References}

Dehant, V. et al., 1999, Celes. Mech., 72, 245.

Dehant, V. and Defraigne, P., 1997, J. Geophys. Res., 102 B, 27, 659.

Getino, J., Ferrándiz, J.M., 1999, Proc. Journées 99, (in press).

Getino, J., Ferrándiz, J.M., 1999, Mon. Not. RAS, 306, L45.

Hori, G., 1966, Publ. Astron. Soc. Japan, 18, 287.

Jeffreys, H. and Vicente, R., 1957, Mon. Not. RAS, 117, 142 and 162.

Mathews, P., Buffett, B., Herring, T., 1999, private communication.

McCarthy, D.(ed), 1996, IERS Conventions.

Sasao, T., Okubo, S. and Saito, M., 1980, IAU Symp. No. 78, 165.

Schastok, J., 1997, Geophys. J. Int., 130, 137. 31, 651. 